\title{
Debilitating Skin Toxicity Associated with Pembrolizumab Therapy in an 81-Year-Old Female with Malignant Melanoma
}

\author{
Muhammad O. Khokhar ${ }^{a} \quad$ Jacob Kettle ${ }^{b}$ Amruth R. Palla \\ ${ }^{a}$ Division of Hematology and Oncology, Department of Medicine, Ellis Fischel Cancer \\ Center, University of Missouri Columbia, Columbia, MO, USA; ${ }^{b}$ Division of Hematology \\ and Oncology, Department of Pharmacy, Ellis Fischel Cancer Center, University of \\ Missouri Columbia, Columbia, MO, USA
}

\section{Keywords}

Pembrolizumab · Immune checkpoint inhibitors · Skin toxicity

\section{Abstract}

Frequently described immune-mediated adverse effects of immune therapy include dermatological complications, hepatitis, colitis, pneumonitis, and endocrinopathies. As utilization of pembrolizumab and related agents continues to expand both in the available indications as well as duration of exposure, there remains a significant potential to uncover previously undescribed adverse events. From a dermatological standpoint, 39\% of patients receiving pembrolizumab therapy experience some form of skin-related drug toxicity [Naidoo et al.: Ann Oncol 2015;26:2375-2391]. We describe a case of pembrolizumab-induced disabling autoimmune ectodermal toxicity.

\section{Introduction}

The important connection between immunology and oncology has been long understood; however, the therapeutic implications were previously limited to the use of cytokines 
in the diseases such as melanoma and renal cell carcinoma. This has shifted dramatically in the recent years with the emergence of immunotherapy utilizing various checkpoint inhibitors. This new group of immune therapy includes CTLA-4 inhibitors (ipilimumab) to PD-1 inhibitors (nivolumab, pembrolizumab) and PD-L1 inhibitors (atezolizumab), as well as numerous other molecules in the development. The clinical utility of these agents include melanoma [1] and renal cell carcinoma [2] as well as numerous malignancies not previously established as responsive to immunologically derived therapy.

While immune checkpoints are pivotal to preventing excessive immune responses directed toward healthy tissue, the malignant cells also utilize these pathways as a mechanism to evade the immune system. The incorporation of checkpoint inhibitors into antineoplastic therapy serves to reactivate the action of the immune system on the cancer. Pembrolizumab, a monoclonal antibody, binds to PD-1 receptor on T cells, thereby preventing the binding of PD-1 receptor to the PD-1 ligand (PD-L1) expressed on tumor cells. This acts to reverse the inhibition of the $\mathrm{T}$ cells to generate an antitumor response and has resulted in pembrolizumab receiving indications including metastatic melanoma, metastatic non-small cell lung cancer, and recurrent or metastatic head and neck cancers. The consequences of this antitumor effect includes onset of numerous immunological complications resulting from the disruption in self-tolerance.

\section{Case Presentation}

Our patient is an 81-year-old female with the past medical history of stage IV melanoma, which was originally diagnosed in 2011. The patient had BRAF-negative disease and had failed multiple past therapies, including temozolamide, ipilimumab, everolimus, sorafenib, and nano-albumin-bound paclitaxel (in this respective order). Patient received about 6 months of therapy with nano-albumin-bound paclitaxel, and repeat imaging showed progression of disease. The patient was then initiated on pembrolizumab at a dose of $2 \mathrm{mg} / \mathrm{kg}$ every 3 weeks immediately following FDA approval in September 2014. After 3 months of therapy, imaging showed responsive disease, with reduced FGD avidity on the PET scan. Therapy was subsequently continued for 28 cycles as clinical and radiographic response was maintained. After completion of 28 cycles of therapy, the patient began to notice discomfort, redness, and inflammation around the eyes. The patient was seen by ophthalmology and was diagnosed with blepharoconjuncitivits and given dexamethasone and moxifloxacin eye drops. Although this event was considered a potential autoimmune complication of pembrolizumab, therapy was continued given her response to treatment and the manageable nature of her condition.

Two weeks following her 29th dose of pembrolizumab, the patient noticed markedly increased redness, edema and hemorrhagic desquamation of her lips (Fig. 1). Further, she demonstrated erosive skin lesions on her fingers and desquamation of the nails (Fig. 2, Fig. 3) and a return of previously described ophthalmological complications with increased severity (Fig. 4). Pain and discomfort resulting from hemorrhagic crusting of her lips and oral mucosa contributed to her poor oral intake and self-care and ultimately necessitated an admission for dehydration. In addition to intravenous fluids, parenteral glucocorticoids (prednisone dosed at $1 \mathrm{mg} / \mathrm{kg}$ ) were initiated, as autoimmune complications from pembrolizumab therapy were the suspected cause of her symptoms.

Dermatology was consulted for additional evaluation. A skin biopsy from the erosion of the thumb showed psoriasiform lichenoid dermatitis with acrosyringeal neutrophils and 
direct immunofluorescence was negative for paraneoplastic pemphigus. These results supported the suspicion that her symptoms were in fact the result of autoimmune complications induced by her pembrolizumab therapy.

Her mucosal and dermal lesions demonstrated marked healing after 5 days of intravenous steroids and she was soon able to maintain adequate oral intake. A slow oral prednisone taper was initiated at discharge (reduced by $10 \mathrm{mg}$ per week) and at approximately 1 month after discharge, she exhibited near complete resolution of her clinical manifestations. CT scan completed 6 weeks after her last dose of pembrolizumab showed no evidence of disease progression compared to a scan from 3 months prior. Given the debilitating nature of her dermal adverse effects and continued disease control, pembrolizumab was held indefinitely.

Having exhausted all previous lines of therapy, a detailed discussion with the patient was held and it was decided to challenge her with nivolumab. It remains to be seen how she will react to nivolumab and if perhaps the autoimmune manifestations are class specific. We will have a very low threshold to discontinue nivolumab with any similar adverse reactions like those mentioned above.

\section{Discussion}

Dermal reactions are amongst the more common known adverse effects induced by checkpoint inhibitors and appear to be more common when these agents are used in treatment of melanoma compared to other malignancies. Although reports indicate perhaps as many as one in three patients may experience skin rash, cutaneous adverse effects such as papular rash, pruritus, and hypopigmentation, and the severity of these events if generally limited [3].

Jour et al. [4] described several cases of bullous skin eruptions associated with the antiepidermal antibodies due to anti-PD-1 antibody therapy. The report consists of patients who presented with vesicles and blisters on the skin while being treated with anti-PD-1 antibody immunotherapy with either nivolumab or pembrolizumab. Four of the five patients studied had evidence of subepidermal bullae with immunohistochemical studies showing antibodies to type IV collagen. Direct immunofluorescence studies in three of the four patients tested showed linear IgG and C3 immune deposits on the blister roof suggesting a diagnosis of bullous pemphigoid. These findings are not consistent with our report, as this patient had negative immunofluorescence on skin biopsy [4].

In a series of 300 consecutive drug eruptions, lichenoid psoriasiform dermatitis was observed in 18 cases (6\%). This includes a patchy lichenoid infiltrate of lymphocytes and uneven psoriasiform epidermal hyperplasia. All of these features can also be seen in the patch stage of mycosis fungoides [5]. Drug eruptions, however, retain signs of acute inflammation, including edema of the papillary dermis and presence of neutrophills in the infiltrate, not seen in the mycosis fungoides. This type of presentation is in agreement with our case.

Interestingly, preliminary data suggests that patients developing skin reactions may experience longer progression-free interval in comparison to patients not experiencing such toxicity [3]. Although certainly not confirmatory, it is nonetheless fascinating in that the patient demonstrating the most severe dermal toxicity to PD-1 therapy is simultaneously among the best responders amongst the population being treated with such therapies. As data matures, the notion of the correlation of response to toxicity may serve not only as a 
Khokhar et al.: Debilitating Skin Toxicity Associated with Pembrolizumab Therapy in an 81-Year-Old Female with Malignant Melanoma

significant comfort to patients dealing with untoward effect of the therapy, but also may act as a surrogate marker for treatment response.

To the best of our knowledge, a case as we describe of disabling autoimmune ectodermal toxicity involving erosive oral mucositis, ulcerative necrosis of the lip, paronychial erosions, and blepharoconjunctivitis has not previously been documented with pembrolizumab or alternative PD-1-targeted therapies. Despite the radical impact it has had on patient care, a tremendous amount of uncertainties remains in the era of immune oncology. When unexpected complications arise for patients on the checkpoint-directed therapy, clinicians must remain ever mindful that an unanticipated autoimmune-mediated complication of therapy may be involved. Early recognition and appropriate management of these adverse effects is important and may prompt initiation of life-saving therapy.

\section{Statement of Ethics}

The patient has approved this case report. Since this is a case report and no animals were mentioned, the compliance with guidelines of human studies and animal welfare regulations and approval by IRB does not apply here.

\section{Disclosure Statement}

None of the authors have any conflicts of interest to disclose. All the authors have read the manuscript and agree to its publication in this form. We assure whoever is concerned that this paper is not under simultaneous consideration by any other publication.

\section{References}

1 Specenier P: Nivolumab in melanoma. Expert Rev Anticancer Ther 2016, DOI: 10.1080/14737140.2016.1249856.

2 Mennitto A, Grassi P, Ratta R, Verzoni E, Prisciandaro M, Procopio G: Nivolumab in the treatment of advanced renal cell carcinoma: clinical trial evidence and experience. Ther Adv Urol 2016;8:319-326.

3 Sanlorenzo M, Vujic I, Daud A, Algazi A, Gubens M, Luna SA, Lin K, Quaglino P, Rappersberger K, OrtizUrda S: Pembrolizumab cutaneous adverse events and their association with disease progression. JAMA Dermatol 2015;151:1206-1212.

-4 Jour G, Glitza IC, Ellis RM, Torres-Cabala CA, Tetzlaff MT, Li JY, Nagarajan P, Huen A, Aung PP, Ivan D, Drucker CR, Prieto VG, Rapini RP, Patel A, Curry JL: Autoimmune dermatologic toxicities from immune checkpoint blockade with anti-PD-1 antibody therapy: a report on bullous skin eruptions. J Cutan Pathol 2016;43:688-696.

5 Weyers W, Metze D: Histopathology of drug eruptions - general criteria, common patterns, and differential diagnosis. Dermatol Pract Concept 2011;1:33-47. 


\section{Case Reports in Oncology}

Khokhar et al.: Debilitating Skin Toxicity Associated with Pembrolizumab Therapy in an 81-Year-Old Female with Malignant Melanoma

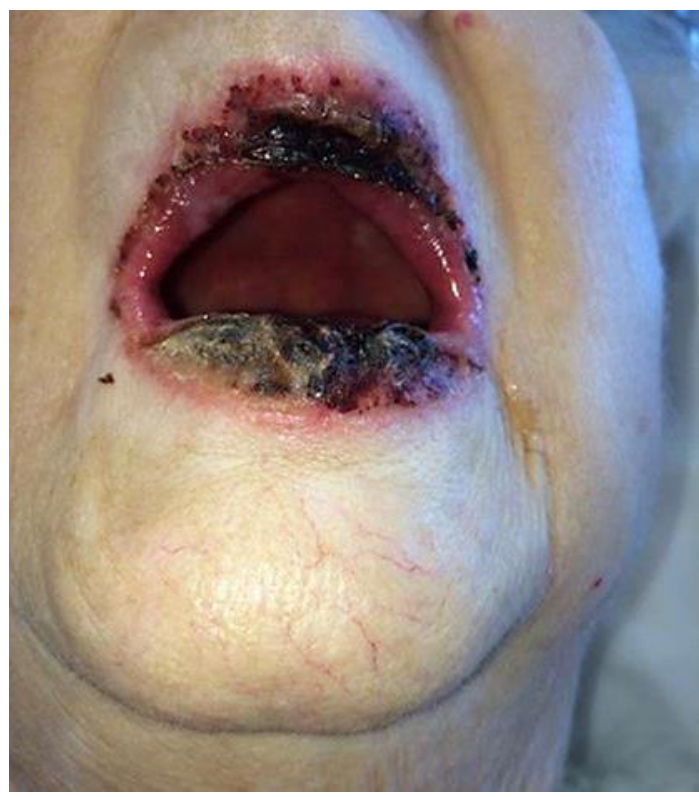

Fig. 1. Hemorrhagic crusting of the lip. 


\section{Case Reports in Oncology}

\begin{tabular}{l|l}
\hline Case Rep Oncol 2016;9:833-839 \\
\hline DOI: 10.1159/000452944 & $\begin{array}{l}\text { C 2016 The Author(s). Published by S. Karger AG, Basel } \\
\text { www.karger.com/cro }\end{array}$ \\
\hline
\end{tabular}

Khokhar et al.: Debilitating Skin Toxicity Associated with Pembrolizumab Therapy in an 81-Year-Old Female with Malignant Melanoma

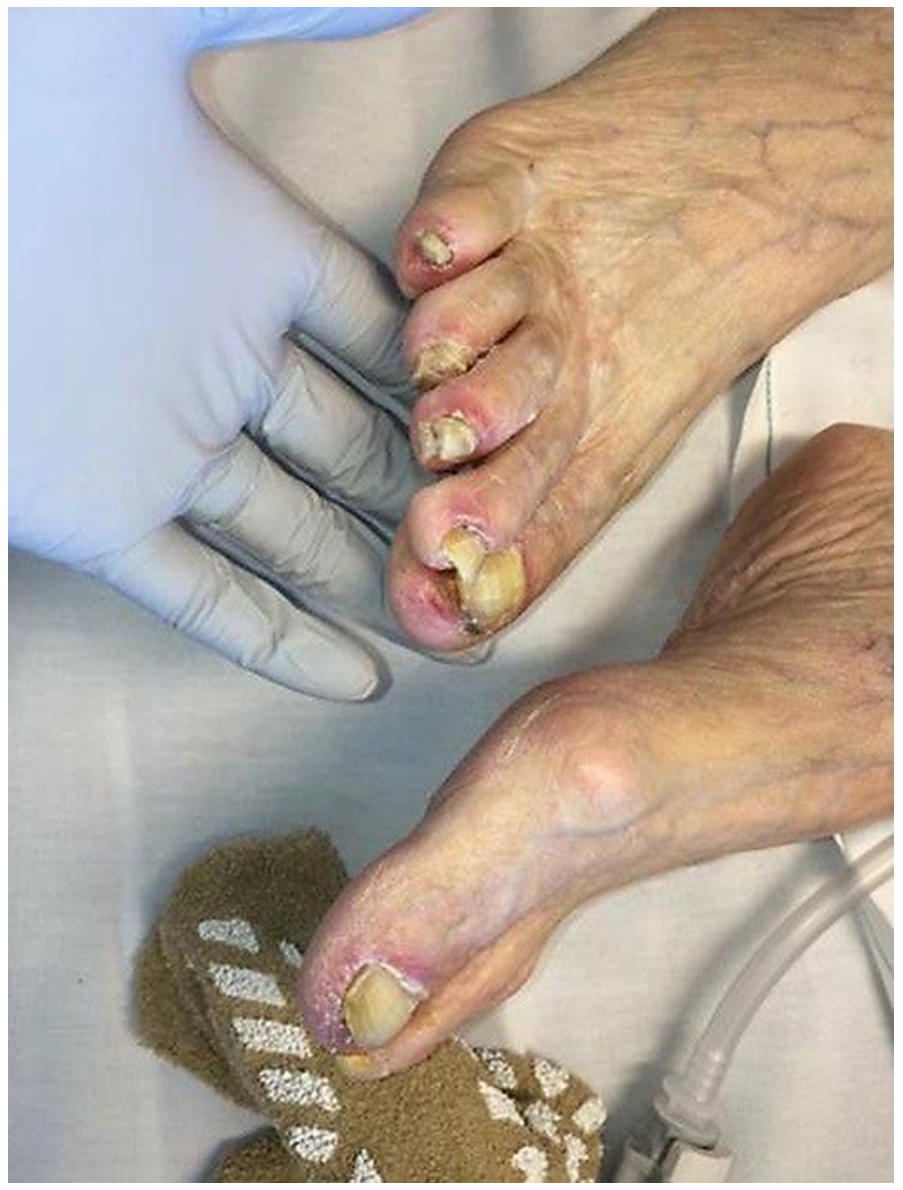

Fig. 2. Toenail toxicity, with peeling of skin and desquamation. 


\section{Case Reports in Oncology}

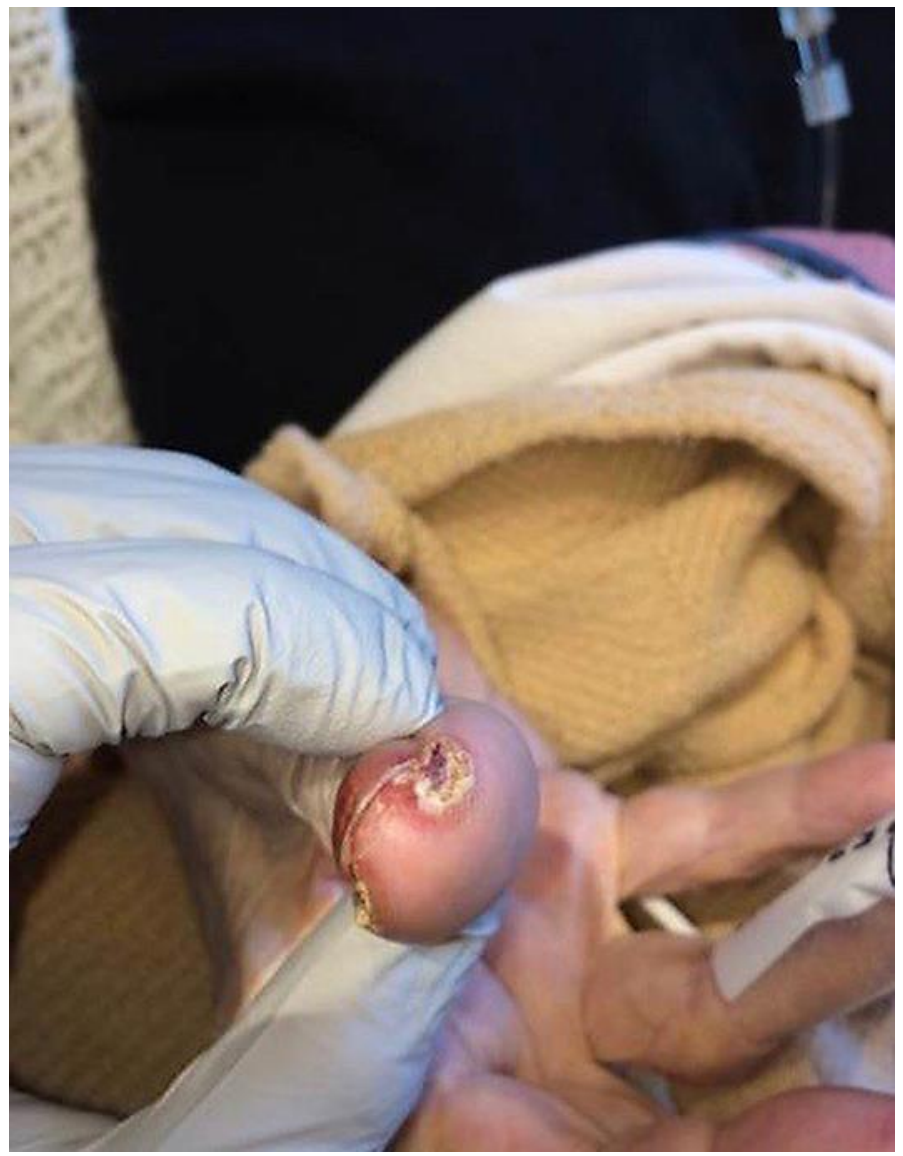

Fig. 3. Fingernail toxicity, with peeling of skin and desquamation.

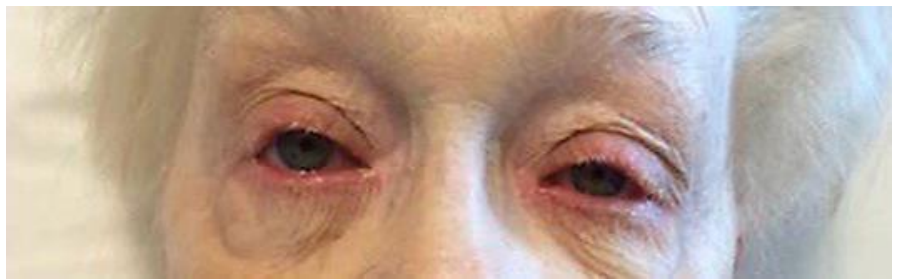

Fig. 4. Blepharoconjunctivitis.

Khokhar et al.: Debilitating Skin Toxicity Associated with Pembrolizumab Therapy in an 81-Year-Old Female with Malignant Melanoma 